\title{
Extraction and purification of B-phycoerythrin from the red microalga Rhodosorus marinus
}

\section{Extracción y purificación de B-ficoeritrina de la microalga roja Rhodosorus marinus}

\author{
GA Básaca-Loya ${ }^{1}$, MA Valdez², EA Enríquez-Guevara ${ }^{1}$, LE Gutiérrez-Millán³ ${ }^{3}$ MG. Burboa ${ }^{3 *}$ \\ ${ }^{1}$ Departamento de Investigación en Polímeros y Materiales, Universidad de Sonora, Blvd. Luis Encinas y Rosales S/N, Col. \\ Centro, A.P. 1819, Hermosillo, CP 83000, Sonora, México. \\ ${ }^{2}$ Departamento de Física y Departamento de Investigación en Polímeros y Materiales, Universidad de Sonora, Blvd. Luis \\ Encinas y Rosales S/N, Col. Centro, Hermosillo, CP 83000, Sonora, México. \\ ${ }^{3}$ Departamento de Investigaciones Científicas y Tecnológicas (DICTUS), Universidad de Sonora, Blvd. Luis Encinas y Rosales \\ S/N, Col. Centro, Hermosillo, CP 83000, Sonora, México.*E-mail: mburboa@correom.uson.mx
}

\begin{abstract}
A description is given of the purification of B-phycoerythrin (B-PE) from the red microalga Rhodosorus marinus. Initially, phycobiliproteins were released from the microalgal cells by manual cellular fragmentation and sonication. B-PE was extracted with ammonium sulfate precipitation, and purified by anionic and size exclusion chromatography. Its purity was tested using indexes and sodium dodecyl sulfate-polyacrylamide gel electrophoresis. Spectroscopic characterization of B-PE was performed by UV-visible spectroscopy, fluorescence spectroscopy, and circular dichroism. Rhodosorus marinus showed three types of phycobiliproteins: phycoerythrin, phycocyanin, and allophycocyanin. The purified B-PE showed a purity ratio $\left(\mathrm{A}_{545} / \mathrm{A}_{280}\right)$ of 4.8 , characteristic peaks at 540 and $562 \mathrm{~nm}$ with a shoulder at $498 \mathrm{~nm}$, fluorescence emission maximum at $578 \mathrm{~nm}$, and a secondary structure almost stable with $\mathrm{pH}$ changes. B-PE was found to be the predominant pigment in $R$. marinus and this red microalga could be a viable option for the recovery of this chromoprotein.
\end{abstract}

Key words: microalgae, phycobiliproteins, phycoerythrin, pigments, Rhodosorus marinus.

\section{Resumen}

En este trabajo se describe la purificación de la proteína B-ficoeritrina (B-PE) de la microalga roja Rhodosorus marinus. Inicialmente se extrajeron las ficobiliproteínas de las células microalgales mediante fragmentación manual y sonicación. La BPE fue purificada mediante precipitación con sulfato de amonio y cromatografía de exclusión por tamaño e intercambio aniónico. Se determinó la pureza de B-PE por medio de índices y electroforesis en gel de poliacrilamida con dodecilsulfato de sodio. La caracterización espectroscópica de B-PE se realizó mediante espectroscopía UV-visible, espectroscopía de fluorescencia y dicroísmo circular. Rhodosorus marinus presentó tres tipos de ficobiliproteínas: ficoeritrina, ficocianina y aloficocianina. La B-PE purificada mostró un índice de pureza $\left(\mathrm{A}_{545} / \mathrm{A}_{280}\right)$ de 4.8, picos característicos a 540 y 562 nm con un hombro a 498 nm, un máximo de emisión de fluorescencia de $578 \mathrm{~nm}$, y una estructura secundaria casi estable ante cambios de $\mathrm{pH}$. La B-PE mostró ser el pigmento predominante en $R$. marinus. Esta microalga roja podría ser una opción viable para la recuperación de esta cromoproteína.

Palabras clave: microalgas, ficobiliproteínas, ficoeritrina, pigmentos, Rhodosorus marinus.

\section{Introduction}

Phycobiliproteins act as photosynthetic accessory pigments in marine algae such as Rhodophyceae, Cyanophyceae, Cryptophyceae, and some Pirrophyceae (Gantt 1975, Ma et al. 2003). They are organized into cellular structures, called phycobilisomes, which are attached in regular arrays to the outer surface of the thylakoids. Phycobiliproteins are water-soluble and composed of $\alpha, \beta$, and $\gamma$ subunits of phycoerythrins (Wang et al. 1998), each of which consists of an apoprotein and one or more kinds of open-chain tetrapyrrole prosthetic groups (chromophores named bilins) that are covalently linked to specific cysteine residues of the apoproteins (Glazer 1981). The spectroscopic properties of individual biliproteins depend mainly on the chemical nature of the bilins they carry (MacColl and

\section{Introducción}

Las ficobiliproteínas actúan como pigmentos accesorios para la fotosíntesis en algas marinas de las Rhodophyceae, Cyanophyceae, Cryptophyceae y algunas Pirrophyceae (Gantt 1975, Ma et al. 2003). Se encuentran organizadas en estructuras celulares, llamadas ficobilisomas, las cuales están unidas en conjuntos regulares a la superficie exterior de los tilacoides. Las ficobiliproteínas son solubles en agua y están compuestas de subunidades $\alpha, \beta$ y $\gamma$ de las ficoeritrinas (Wang et al. 1998), cada una de las cuales consiste de una apoproteína y uno o más tipos de grupos prostéticos tetrapirrólicos de cadena abierta (cromóoros llamados bilinas) unidos mediante enlaces covalentes a los residuos específicos de cisteína de las apoproteínas (Glazer 1981). Las propiedades espectroscópicas de las 
Guard-Friar 1983, Glazer 1989, Grossman 1990, Holzwarth 1991, Moerschel 1991). Phycobiliproteins are divided into three classes based on their absorption properties: phycoerythrins $\left(\lambda_{\max } \sim 540-570 \mathrm{~nm}\right)$, which have the chromophores phycoerythrobilin and phycourobilin; phycocyanins $\left(\lambda_{\max } \sim\right.$ $610-620 \mathrm{~nm}$ ), which contain either a mixture of the phycocyanobilin and phycoerythrobilin chromophores or just phycocyanobilin, depending on the species of origin; and allophycocyanins $\left(\lambda_{\max } \sim 650-655 \mathrm{~nm}\right)$, with phycocyanobilin as prosthetic group (Glazer 1984, Bermejo et al. 2003). The chromophores confer a characteristic color to each phycobiliprotein: red to phycoerithrin, bright blue to phycocyanin, and green-blue to allophycocyanin. The major classes of phycoerythrins have been reported to differ in their absorption characteristics: B-phycoerythrins show $\lambda_{\max } \sim 565,546 \mathrm{~nm}$ and a shoulder at $\sim 499 \mathrm{~nm}$; R-phycoerythrins show $\lambda_{\max } \sim 568,499$ $\mathrm{nm}$ and a shoulder at $\sim 545 \mathrm{~nm}$; and C-phycoerythrins show $\lambda_{\max } \sim 565 \mathrm{~nm}$ (Hilditch et al. 1991, D'Agnolo et al. 1994, Bermejo et al. 2001).

Among the phycobiliproteins, B-phycoerythrin (B-PE) is a major light-harvesting pigment of red microalgae, and it is the most valuable of the three main classes of phycoerythrins (B, $\mathrm{R}$, and $\mathrm{C}$ ) due to its high fluorescence efficiency and its intense and unique pink color. It is thus widely used as a fluorescent probe and analytical reagent, as well as being employed as a natural dye in foodstuffs and cosmetics (Bermejo et al. 2003) and in the development of biosensors (Martek Corporation, http://www.marketbio.com) (Ayyagari et al. 1995, Haugland 1996, Bermejo et al. 2002, Benavides and Rito-Palomares 2004). Phycobiliproteins have also been shown to have therapeutic value due to their immunomodulating and anticarcinogenic activities (Iijima and Shimamatsu 1982).

To date, very different methodologies have been proposed for purifying phycobiliproteins from microalgae. Pure phycobiliproteins from crude algal extracts are usually obtained by a combination of different chromatographic methods and ammonium sulfate precipitation (Gray and Gantt 1975, Grabowski and Gantt 1978, Duerring et al. 1991, Ficner et al. 1992, Bermejo et al. 1997, Tchernov et al. 1999). Most of the proposed methods can be divided into two steps: first, the extraction of phycobiliproteins from the biomass and second, the purification of the extract obtained. The original study conducted by Glazer and Hixson (1975) on B-PE purification consisted of a three chromatographic step scheme, which has since been simplified to two chromatographic steps (Yu et al. 1981, Schoelember et al. 1983, Bermejo et al. 2003, Rossano et al. 2003). In the present study, we used different methods for the extraction of proteins, and anionic and size-exclusion chromatography for their purification.

Porphyridium cruentum is a well-studied red microalga and used for the recovery of B-PE (Schoelember et al. 1983; Hilditch et al. 1991; D'Agnolo et al. 1994; Bermejo et al. 2001, 2002, 2003; Ma et al. 2003; Rossano et al. 2003; Benavides and Rito-Palomares 2004). In this study we describe the B-PE from Rhodosorus marinus, an alga that shows biliproteínas individuales dependen principalmente de la naturaleza química de sus bilinas (MacColl y Guard-Friar 1983, Glazer 1989, Grossman 1990, Holzwarth 1991, Moerschel 1991). Las ficobiliproteínas se dividen en tres clases según sus propiedades de absorción: ficoeritrinas $\left(\lambda_{\max } \sim 540-570 \mathrm{~nm}\right)$, las cuales contienen los cromóforos ficoeritrobilina y ficourobilina; ficocianinas $\left(\lambda_{\max } \sim 610-620 \mathrm{~nm}\right.$ ), que contienen ya sea una mezcla de los cromóforos ficocianobilina y ficoeritrobilina o sólo ficocianobilina, según la especie de origen; y aloficocianinas $\left(\lambda_{\max } \sim 650-655 \mathrm{~nm}\right)$, con ficocianobilina como grupo prostético (Glazer 1984, Bermejo et al. 2003). Los cromóforos dan un color característico a cada ficobiliproteína: rojo a la ficoeritrina, azul brillante a la ficocianina, y verde azulado a la aloficocianina. Se ha encontrado que las principales clases de ficoeritrinas difieren en sus características de absorción: las B-ficoeritrinas muestran $\lambda_{\max } \sim 565$ y $546 \mathrm{~nm}$, con un hombro a $\sim 499 \mathrm{~nm}$; las R-ficoeritrinas $\lambda_{\max } \sim 568$ y 499 $\mathrm{nm}$, con un hombro a $\sim 545 \mathrm{~nm}$; y las C-ficoeritrinas $\lambda_{\max } \sim 565$ nm (Hilditch et al. 1991, D'Agnolo et al. 1994, Bermejo et al. 2001).

Entre las ficobiliproteínas, la B-ficoeritrina (B-PE) es un importante pigmento para la captación de luz por las microalgas rojas, y es la más valiosa de las tres clases de ficoeritrinas (B, R y C) debido a su gran eficiencia de fluorescencia y su intenso y particular color rosa. Por lo tanto, es ampliamente utilizada como una sonda fluorescente y reactivo analítico, además de emplearse como un tinte natural en alimentos y cosméticos (Bermejo et al. 2003), así como para desarrollar biosensores (Martek Corporation, http://www.marketbio.com) (Ayyagari et al. 1995, Haugland 1996, Bermejo et al. 2002, Benavides y Rito-Palomares 2004). También se ha mostrado que las ficobiliproteínas poseen un valor terapeútico debido a sus actividad inmunomoduladora y anticarcinogénica (Iijima y Shimamatsu 1982).

A la fecha, se han propuesto métodos muy diferentes para la purificación de las ficobiliproteínas de microalgas. Generalmente se obtienen ficobiliproteínas puras de extractos crudos de algas mediante una combinación de diversos métodos cromatográficos y la precipitación con sulfato de amonio (Gray y Gantt 1975, Grabowski y Gantt 1978, Duerring et al. 1991, Ficner et al. 1992, Bermejo et al. 1997, Tchernov et al. 1999). La mayoría de los métodos propuestos consta de dos pasos: primero se extraen las ficobiliproteínas de la biomasa y después se purifica el extracto obtenido. El estudio original realizado por Glazer y Hixson (1975) sobre la purificación de la B-PE consistió en un esquema de tres pasos cromatográficos, el cual ya ha sido simplificado a dos pasos (Yu et al. 1981, Schoelember et al. 1983, Bermejo et al. 2003, Rossano et al. 2003). En el presente trabajo se utilizaron diferentes métodos para la extracción de proteínas, y posteriormente cromatografía de exclusión por tamaño e intercambio aniónico para su purificación.

Porphyridium cruentum es una microalga roja que ha sido bien estudiada y utilizada para la obtención de B-PE (Schoelember et al. 1983; Hilditch et al. 1991; D’Agnolo et al. 
potential for the biotechnological production of this protein. It is a cosmopolitan species and represents a very interesting case since the industrial and commercial value of this product is considerable. Notwithstanding, until now, there are no scientific reports known on B-PE from $R$. marinus.

\section{Material and methods}

\section{Algal strain and cultivation conditions}

The study was carried out using the red microalga $R$. marinus provided by UTEX (1723). Microalgae were grown in batch cultures (10-mL culture tubes, 250-mL Erlenmeyer flask, and 18-L carboys) for 25 days, under the following conditions: Ersdchreiber culture medium (Rosowski and Parker 1971), continuous bubbling of air until the 12 -L level, $25^{\circ} \mathrm{C}$, lateral illumination with cool-white fluorescent lamps at a photon flux density of $215 \mu \mathrm{E} \mathrm{m} \mathrm{m}^{-2} \mathrm{~s}^{-1}$ measured at the surface of $250-\mathrm{mL}$ flasks, and a circadian 12-h light and 12-h dark cycle. Microalgae were decanted and harvested by centrifugation at $3000 \mathrm{rpm}$ for 5 min (Marathon 8K Fisher Scientific). See Básaca-Loya et al. (2008).

\section{Preparation and fractionation of the crude extract}

Algal cells ( $4 \mathrm{~g}$ dry biomass) were rinsed with $0.01 \mathrm{M} \mathrm{Na}-$ phosphate buffer containing $0.1 \mathrm{M} \mathrm{NaCl}$ (pH 7.0) (Rossano et al. 2003) and resuspended in the $0.01 \mathrm{M} \mathrm{Na-phosphate/ \textrm {NaCl }}$ buffer $(\mathrm{pH} 7.0)$. The suspension was then frozen at $-20^{\circ} \mathrm{C}$, thawed, sonicated for $30 \mathrm{~min}$, and cellular fragmentation was performed manually in a ceramic pot. Cell debris removal was achieved by centrifugation at $5000 \mathrm{rpm}$, at $4^{\circ} \mathrm{C}$, for $10 \mathrm{~min}$. The supernatant was referred to as crude extract and the precipitate was discarded. The crude extract obtained was saturated with $40 \%$ ammonium sulfate, agitated for $3.5 \mathrm{~h}$ and left at $4^{\circ} \mathrm{C}$ until precipitation, and then centrifuged at $3000 \mathrm{rpm}$ for 10 min. The precipitate was discarded. The supernatant was saturated with $60 \%$ ammonium sulfate (Bermejo et al. 2002), agitated for $15 \mathrm{~h}$ and left at $4^{\circ} \mathrm{C}$ until precipitation, and then centrifuged at $3000 \mathrm{rpm}$ for $10 \mathrm{~min}$. The precipitate was resuspended in the $0.01 \mathrm{M} \mathrm{Na}$-phosphate/ $\mathrm{NaCl}$ buffer $(\mathrm{pH} 7.0)$.

\section{Chromatography}

The remaining ammonium sulfate was removed by chromatography on a Sephadex G-25 (Sigma) column preequilibrated with $0.01 \mathrm{M}$ phosphate buffer $(\mathrm{pH}$ 7.0) containing $0.1 \mathrm{M} \mathrm{NaCl}$. The eluate was then monitored at $280 \mathrm{~nm}$ and collected in 4-mL fractions. The fractions with protein were pooled and applied to an anionic chromatographic column HiPrep 16/10 Q XL $(0.75 \times 10 \mathrm{~cm})$ (Amersham BioSciences), which was pre-equilibrated with $0.01 \mathrm{M}$ phosphate buffer (pH 7.0) containing $0.1 \mathrm{M} \mathrm{NaCl}$.

After washing with $40 \mathrm{~mL}$ of $0.01 \mathrm{M} \mathrm{Na-phosphate/ \textrm {NaCl }}$ buffer ( $\mathrm{pH}$ 7.0), the column was eluted with $0.01 \mathrm{mM}$
1994; Bermejo et al. 2001, 2002, 2003; Ma et al. 2003; Rossano et al. 2003; Benavides y Rito-Palomares 2004). En este estudio se describe la B-PE de Rhodosorus marinus, un alga que muestra potencial para la producción biotecnológica de esta proteína. Es una especie cosmopolita, y de gran interés ya que el valor industrial y comercial de este producto es considerable; no obstante, hasta ahora, no se cuenta con ningún informe científico sobre la B-PE de $R$. marinus.

\section{Materiales y métodos}

\section{Cepa algal y condiciones de cultivo}

En este trabajo se utilizó la microalga roja $R$. marinus proporcionada por UTEX (1723). Las microalgas fueron cultivadas por lotes (tubos de ensayo de $10 \mathrm{~mL}$, frascos Erlenmeyer de $250 \mathrm{~mL}$ y garrafones de $18 \mathrm{~L}$ ) durante 25 días, bajo las siguientes condiciones: medio de cultivo Ersdchreiber (Rosowski y Parker 1971), burbujeo continuo de aire hasta el nivel de $12 \mathrm{~L}, 25^{\circ} \mathrm{C}$, iluminación lateral con lámparas fluorescentes (blanco frío) a una densidad del flujo de fotones de $215 \mu \mathrm{E} \mathrm{m}^{-2} \mathrm{~s}^{-1}$ medida en la superficie de los frascos de 250 $\mathrm{mL}$, y un ciclo circadiano de $12 \mathrm{~h}$ de luz y $12 \mathrm{~h}$ de oscuridad. Las microalgas fueron decantadas y cosechadas mediante centrifugación a $3000 \mathrm{rpm}$ por $5 \mathrm{~min}$ (Marathon 8K Fisher Scientific). Ver el trabajo de Básaca-Loya et al. (2008).

\section{Preparación y fraccionación del extracto crudo}

Las células algales ( $4 \mathrm{~g}$ de biomasa seca) se enjuagaron con amortiguador de fosfato de sodio $0.01 \mathrm{M}$ que contenía $\mathrm{NaCl}$ $0.1 \mathrm{M}$ (pH 7.0) (Rossano et al. 2003) y se resuspendieron en el amortiguador de fosfato de sodio/ $\mathrm{NaCl} 0.01 \mathrm{M}(\mathrm{pH} 7.0)$. $\mathrm{La}$ suspensión fue congelada a $-20^{\circ} \mathrm{C}$, descongelada y sonicada durante $30 \mathrm{~min}$, realizándose la fragmentación celular de forma manual en una olla de cerámica. La eliminación de restos celulares se logró mediante centrifugación a $5000 \mathrm{rpm}$ a $4^{\circ} \mathrm{C}$ por $10 \mathrm{~min}$. El sobrenadante se consideró el extracto crudo y el precipitado se descartó. El extracto crudo obtenido se saturó con sulfato de amonio al $40 \%$, se agitó durante 3.5 h y se dejó reposar a $4{ }^{\circ} \mathrm{C}$ hasta su precipitación; posteriormente se centrifugó a $3000 \mathrm{rpm}$ por $10 \mathrm{~min}$. Se descartó el precipitado. El sobrenadante se saturó con sulfato de amonio al 60\% (Bermejo et al. 2002), se agitó durante $15 \mathrm{~h}$, se dejó reposar a $4^{\circ} \mathrm{C}$ hasta su precipitación, y luego se centrifugó a $3000 \mathrm{rpm}$ por $10 \mathrm{~min}$. El precipitado se resuspendió en amortiguador de fosfato de sodio/ $\mathrm{NaCl} 0.01 \mathrm{M}$ (pH 7.0).

\section{Cromatografía}

El sulfato de amonio restante se eliminó mediante cromatografía en una columna Sephadex G-25 (Sigma) preequilibrada con amortiguador de fosfato $0.01 \mathrm{M}(\mathrm{pH} 7.0)$ que contenía $\mathrm{NaCl} 0.1 \mathrm{M}$. La elución fue monitoreada a $280 \mathrm{~nm}$ y recolectada en fracciones de $4 \mathrm{~mL}$. Las fracciones proteicas se 
phosphate buffer containing $0.1 \mathrm{M}$ and $0.5 \mathrm{M} \mathrm{NaCl}$. The eluate was collected in 4-mL fractions and analyzed. The fractions were pooled and separated in a HPLC system (Agilent 1100) with a Zorbax GF-250 (Agilent) column $(0.94 \times 25 \mathrm{~cm}, 4 \mu \mathrm{m})$, pre-equilibrated with $0.01 \mathrm{M}$ phosphate buffer ( $\mathrm{pH}$ 7.0) containing $0.1 \mathrm{M} \mathrm{NaCl}$. The column was eluted with the $0.01 \mathrm{M}$ Na-phosphate/ $\mathrm{NaCl}$ buffer ( $\mathrm{pH}$ 7.0) and the eluate was collected in 1-mL fractions.

\section{Sodium dodecyl sulfate-polyacrylamide gel electrophoresis (SDS-PAGE)}

Electrophoresis was carried out in a vertical slab gel apparatus (Miniprotean III, Bio-Rad), using a Laemmli buffer system (Laemmli 1970) consisting of a $16.5 \%(\mathrm{w} / \mathrm{v})$ polyacrylamide slab gel, $1.0 \mathrm{~mm}$ thick, containing $0.1 \%(\mathrm{w} / \mathrm{v})$ SDS with a stacking gel of $4 \%$ polyacrylamide. Samples were preincubated with $2 \%(\mathrm{w} / \mathrm{v})$ SDS, $10 \%(\mathrm{v} / \mathrm{v})$ glycerol, $4.5 \%(\mathrm{v} / \mathrm{v})$ $\beta$-mercaptoethanol, $0.025 \%(\mathrm{w} / \mathrm{v})$ bromophenol blue, and $60 \mathrm{mM}$ Tris- $\mathrm{HCl}$ buffer (pH 6.8) for $5 \mathrm{~min}$ at $95^{\circ} \mathrm{C}$. Gels were run at room temperature and visualized by staining with $0.1 \%$ (w/v) Coomassie brilliant blue R-250, 40\% (v/v) methanol, and $7 \%(\mathrm{v} / \mathrm{v})$ acetic acid for $30 \mathrm{~min}$, and destained in dilute acetic acid. The following proteins were used as molecular mass markers: phosphorilase $b(94000)$, albumin $(67000)$, ovalbumin $(43000)$, carbonic anhydrase (30 000), trypsin inhibitor (20 100), and $\alpha$-lactalbumin (14 400).

\section{Spectroscopic measurements}

Absorption spectra were recorded on a Beckman Coulter UV/VIS DU 530 spectrophotometer with a 1-cm light path. The scan wavelength was $200-900 \mathrm{~nm}$. Protein concentrations for these experiments ranged from 0.1 to $0.5 \mathrm{~g} \mathrm{~L}^{-1}$. Fluorescence emission spectra were recorded at room temperature by a Perkin Elmer LS-50B luminescence spectrophotometer with an excitation wavelength of $545 \mathrm{~nm}$. Protein concentrations were chosen so that reabsorption of the emission was negligible.

Circular dichroism spectra were recorded on a Jasco (Tokyo, Japan) J-810 spectropolarimeter, using 1-mm path length quartz cells for measurements in the far UV region $(180-260 \mathrm{~nm})$. The protein concentration of the fractions obtained by anionic chromatography was $0.2 \mathrm{mg} \mathrm{mL}^{-1}$. A $10 \mathrm{mM}$ Na-phosphate buffer ( $\mathrm{pH} 2$ and $\mathrm{pH}$ 7) was used and the $\mathrm{pH}$ was adjusted with diluted hydrochloric acid. The secondary structure was analyzed with the DICHROWEB software (http://www.cryst.bbk.ac.uk/cdweb/html/home.html) and the molecular information of B-PE was obtained from Bermejo et al. (2001). All spectra were recorded at room temperature.

\section{Analytical procedures}

Protein concentration in the samples was estimated by the Bradford method (Bradford 1976). The purity of B-PE was determined as the relation between absorbances at 545 and 280 juntaron y se introdujeron en una columna de intercambio aniónico HiPrep 16/10 Q XL $(0.75 \times 10 \mathrm{~cm})$ (Amersham BioSciences), equilibrada previamente con amortiguador de fosfato $0.01 \mathrm{M}(\mathrm{pH} 7.0)$ con $\mathrm{NaCl} 0.1 \mathrm{M}$.

Después de enjuagar con $40 \mathrm{~mL}$ de amortiguador de fosfato de sodio/ $\mathrm{NaCl} 0.01 \mathrm{M}(\mathrm{pH} 7.0)$, la columna se enjuagó con amortiguador de fosfato $0.01 \mathrm{mM}$ que contenía $\mathrm{NaCl} 0.5 \mathrm{M}$ y $0.1 \mathrm{M}$. Esta elución fue recolectada en fracciones de $4 \mathrm{~mL} \mathrm{y}$ analizada. Las fracciones se juntaron y luego se separaron en un sistema (Agilent 1100) de cromatografía líquida de alta resolución (HPLC por sus siglas en inglés) con una columna Zorbax GF-250 (Agilent, $0.94 \times 25 \mathrm{~cm}, 4 \mu \mathrm{m}$ ), equilibrada previamente con amortiguador de fosfato $0.01 \mathrm{M}(\mathrm{pH}$ 7.0) con $\mathrm{NaCl} 0.1 \mathrm{M}$. La columna se enjuagó con amortiguador de fosfato de sodio/ $\mathrm{NaCl} 0.01 \mathrm{M}(\mathrm{pH} 7.0)$ y la elución se recolectó en fracciones de $1 \mathrm{~mL}$.

\section{Electroforesis en gel de poliacrilamida con dodecilsulfato de sodio (SDS-PAGE por sus siglas en inglés)}

La electroforesis vertical se realizó en un aparato Miniprotean III (Bio-Rad), usando un sistema de amortiguador discontinuo (Laemmli 1970), el cual consiste en un gel de poliacrilamida al $16.5 \%(\mathrm{p} / \mathrm{v})$ de $1.0 \mathrm{~mm}$ de grosor que contiene SDS al $0.1 \%(\mathrm{p} / \mathrm{v})$, con un gel concentrador de poliacrilamida al $4 \%$. Las muestras fueron previamente incubadas con SDS al $2 \%(\mathrm{p} / \mathrm{v})$, glicerol al $10 \%(\mathrm{v} / \mathrm{v}), \beta$-mercaptoetanol al $4.5 \%(\mathrm{v} / \mathrm{v})$, azul de bromofenol al $0.025 \%(\mathrm{p} / \mathrm{v})$ y amortiguador Tris- $\mathrm{HCl} 60 \mathrm{mM}(\mathrm{pH} 6.8)$ durante $5 \mathrm{~min}$ a $95^{\circ} \mathrm{C}$. Los geles se incubaron a temperatura ambiente y se visualizaron mediante tinción con azul brillante de Coomassie R-250 al $0.1 \%(\mathrm{p} / \mathrm{v})$, metanol al $40 \%(\mathrm{v} / \mathrm{v})$ y ácido acético al 7\% (v/v) por $30 \mathrm{~min}$; se destiñeron en ácido acético diluido. Se emplearon las siguientes proteínas como marcadores de peso molecular: fosforilasa $b(94000)$, albúmina (67 000), ovalbúmina (43 000), anhidrasa carbónica (30 000), inhibidor de tripsina (20 100) y $\alpha$-lactalbúmina (14 400).

\section{Mediciones espectroscópicas}

Se registraron espectros de absorción en un espectrofotómetro UV-VIS DU 530 (Beckman Coulter) con una trayectoria óptica de $1 \mathrm{~cm}$. La longitud de onda fue de 200 a $900 \mathrm{~nm}$. Las concentraciones de proteínas en estos ensayos variaron entre 0.1 y $0.5 \mathrm{~g} \mathrm{~L}^{-1}$. Los espectros de emisión de fluorescencia se registraron a temperatura ambiente usando un espectrofotómetro de luminiscencia (Perkin Elmer LS-50B) con una longitud de onda de excitación de $545 \mathrm{~nm}$. Las concentraciones proteicas se escogieron para que la reabsorción de la emisión fuese insignificante.

Los espectros de dicró́smo cicular se registraron con un espectropolarímetro Jasco (modelo J-810, Tokio, Japón), usando células de cuarzo con una trayectoria óptica de $1 \mathrm{~mm}$ para las mediciones en la región del UV lejano (180-260 nm). La concentración de proteína en las fracciones obtenidas 
$\mathrm{nm}$ (i.e., purity of B-PE $=\mathrm{A}_{545} / \mathrm{A}_{280}$ ) (Bermejo et al. 2001), since the absorption spectrum of this protein exhibits a peak at $545 \mathrm{~nm}$.

\section{Results}

We obtained $145 \mathrm{~mL}$ of crude extract from the microalga culture. The analysis indicated that the protein content was $1.6372 \mathrm{mg} \mathrm{mL}^{-1}$. The absorption spectrum of the crude extract is shown in figure 1. In this spectrum, three peaks corresponded to the B-PE maxima (562 and $540 \mathrm{~nm}$, with a shoulder at $498 \mathrm{~nm}$ ), one peak corresponded to the R-phycocyanin maximum $(620 \mathrm{~nm})$, and another peak was observed for allophycocyanin $(650 \mathrm{~nm})$. This shows that R-phycocyanin and allophycocyanin contents are only minor components of the total phycobilins of these cells; the bulk consists of B-PE.

The precipitate obtained after $60 \%$ ammonium sulfate saturation was resuspended in $30 \mathrm{~mL}$ of $0.01 \mathrm{M} \mathrm{Na}$-phosphate buffer. The protein concentration was $2.445 \mathrm{mg} \mathrm{mL}^{-1}$. This solution was desalted using Sephadex G-25 (Sigma) and the fractions obtained were applied to a Q Sepharose column. In some experiments we used different buffers for elution in anionic chromatography: a discontinuous gradient with $0.01 \mathrm{M}$ Na-phosphate/0.1 M NaCl (pH 7) and 0.05 M Na-phosphate/ $0.1 \mathrm{M} \mathrm{NaCl}$ was not found to yield satisfactory results. Better results were found using a discontinuous gradient with different concentrations of $\mathrm{NaCl}$ in $0.01 \mathrm{M} \mathrm{Na}$-phosphate ( $\mathrm{pH} 7.0$ ): $0.1 \mathrm{M}, 0.25$ and $0.5 \mathrm{M} \mathrm{NaCl}$ and the majority of B-PE and Rphycocyanin in the phycobiliproteins were eluted with $0.01 \mathrm{M}$ Na-phosphate $/ 0.1 \mathrm{M} \mathrm{NaCl}$ (fig. 2). Therefore, the results showed that an ionic strength was enough for separation of the phycoerythrin. The absorbance at $280 \mathrm{~nm}$ (most proteins

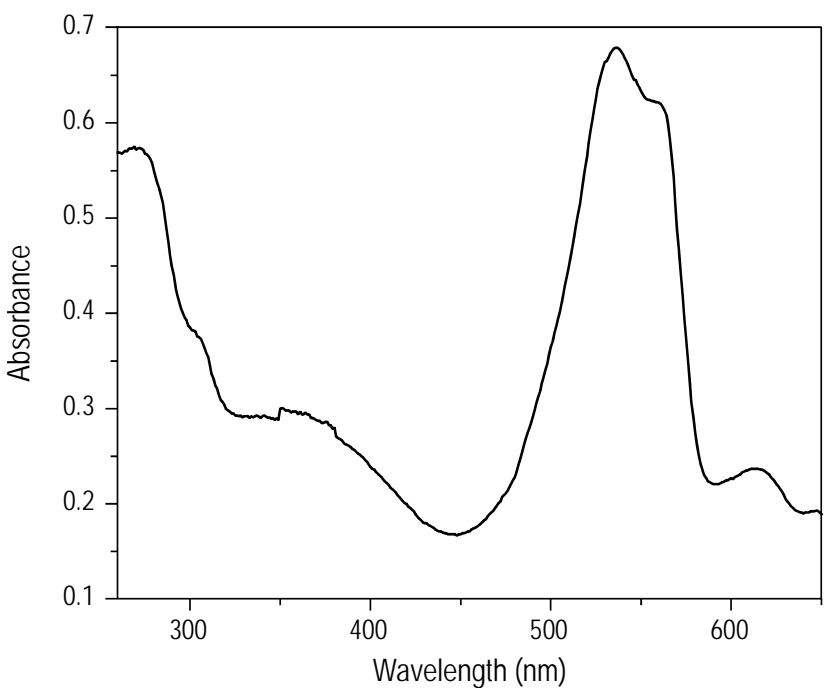

Figure 1 Absorption spectrum of crude extract obtained from Rhodosorus marinus biomass.

Figura 1 Espectro de absorción del extracto crudo obtenido de la biomasa de Rhodosorus marinus. mediante cromatografía de intercambio aniónico fue de $0.2 \mathrm{mg}$ $\mathrm{mL}^{-1}$. Se usó un amortiguador de fosfato de sodio $10 \mathrm{mM}(\mathrm{pH}$ 2 y pH 7) y el pH se ajustó con ácido clorhídrico diluido. La estructura secundaria se analizó utilizando la paquetería DICHROWEB (http://www.cryst.bbk.ac.uk/cdweb/html/ home.html) y la información molecular en relación a la B-PE se obtuvo de Bermejo et al. (2001). Todos los espectros se registraron a temperatura ambiente.

\section{Procedimientos analíticos}

La concentración de proteína en las muestras se estimó por el método de Bradford (1976). La pureza de B-PE fue determinada como la relación entre las absorbancias a 545 y $280 \mathrm{~nm}$ (i.e., pureza de $\mathrm{B}-\mathrm{PE}=\mathrm{A}_{545} / \mathrm{A}_{280}$ ) (Bermejo et al. 2001), ya que el espectro de absorción de esta proteína presenta un pico a $545 \mathrm{~nm}$.

\section{Resultados}

Se obtuvieron $145 \mathrm{~mL}$ de extracto crudo de los cultivos microalgales. El análisis mostró que el contenido de proteína fue $1.6372 \mathrm{mg} \mathrm{mL}^{-1}$. En la figura 1 se muestra el espectro de absorción del extracto crudo. En tal espectro, tres picos correspondieron a los máximos de B-PE (562 y $540 \mathrm{~nm}$, con un hombro a $498 \mathrm{~nm}$ ), un pico correspondió al máximo de Rficocianina $(620 \mathrm{~nm})$ y otro correspondió a la aloficocianina $(650 \mathrm{~nm})$. Esto indica que los contenidos de R-ficocianina y aloficocianina son componentes menores del total de ficobilinas en estas células, siendo B-PE el pigmento predominante.

El precipitado obtenido después de la saturación con sulfato de amonio al $60 \%$ fue resuspendido en $30 \mathrm{~mL}$ de amortiguador de fosfato de sodio $0.01 \mathrm{M}$. La concentración de proteínas entonces fue $2.445 \mathrm{mg} \mathrm{mL}^{-1}$. Esta solución se desalinizó en una columna Sephadex G-25 (Sigma) y las fracciones obtenidas se introdujeron en una columna Sepharose Q. En algunos ensayos se utilizaron diferentes amortiguadores para la elución en la cromatografía aniónica: un gradiente discontinuo con fosfato de sodio $0.01 \mathrm{M} / \mathrm{NaCl} 0.1 \mathrm{M}(\mathrm{pH}$ 7) y fosfato de sodio $0.05 \mathrm{M} / \mathrm{NaCl} 0.1 \mathrm{M}$ no produjo resultados satisfactorios. Se obtuvieron mejores resultados usando un gradiente discontinuo con diferentes concentraciones de $\mathrm{NaCl}$ en fosfato de sodio $0.01 \mathrm{M}$ (pH 7.0): la elución de $\mathrm{NaCl} 0.1,0.25$ y $0.5 \mathrm{M}$ y la mayor parte de B-PE y R-ficocianina en las ficobiliproteínas se obtuvo con fosfato de sodio $0.01 \mathrm{M} / \mathrm{NaCl} 0.1 \mathrm{M}$ (fig. 2). Por lo tanto, los resultados mostraron que la fuerza iónica fue suficiente para la separación de la ficoeritrina. La absorbancia a $280 \mathrm{~nm}$ (la mayoría de las proteínas aborben a $280 \mathrm{~nm}$ ) disminuyó notablemente en relación con el extracto crudo, lo que indica una purificación considerable de la ficobilina en comparación con otras proteínas celulares, mientras que los máximos de B-PE (540 y $562 \mathrm{~nm}$ ) fueron los componentes principales. También se observó que la elución con fosfato $0.01 \mathrm{M} / \mathrm{NaCl}$ $0.5 \mathrm{M}$ en la cromatografía de intercambio aniónico produjo BPE; sin embargo, se obtuvieron bajas concentraciones de proteínas con menor pureza. 


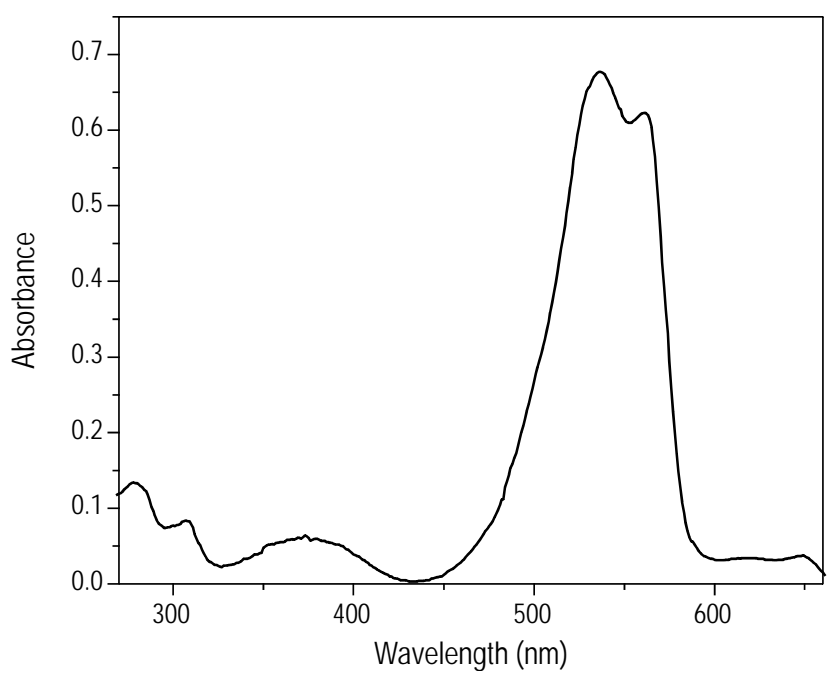

Figure 2. Absorption spectrum obtained from the anionic chromatography (Q Sheparose), using 0.01 M Na-phosphate/0.1 M NaCl buffer ( $\mathrm{pH} 7.0$ ).

Figura 2. Espectro de absorción obtenido mediante cromatografía de intercambio aniónico (Q Sheparose), utilizando fosfato de sodio $0.01 \mathrm{M} /$ $\mathrm{NaCl} 0.1 \mathrm{M}(\mathrm{pH} 7.0)$ como amortiguador.

absorb at $280 \mathrm{~nm}$ ) greatly decreased relative to the crude extract, indicating considerable purification of the phycobilin compared with other extraneous proteins, whereas B-PE peaks $(540,562 \mathrm{~nm})$ were the main components. We also observed that elution with $0.01 \mathrm{M}$ phosphate $/ 0.5 \mathrm{M} \mathrm{NaCl}$ on anionic chromatography produced B-PE, but low concentrations of protein with lower purity were obtained.

The fraction pool obtained using the elution with $0.01 \mathrm{M}$ phosphate $/ 0.1 \mathrm{M} \mathrm{NaCl}$ on anionic chromatography was applied to a column for size-exclusion chromatography. The results obtained by HPLC showed a protein with a purity of $4.8\left(\mathrm{~A}_{545} /\right.$ $\mathrm{A}_{280}$ ), suitable for the purification steps (fig. 3). The ratio of absorption value at $545 \mathrm{~nm}$ to that at $498 \mathrm{~nm}\left(\mathrm{~A}_{545} / \mathrm{A}_{498}\right)$ was 2.5 ; this value was acceptable for the identification of the phycoerythrin pigment. About $1.292 \mathrm{mg}$ of B-PE was obtained from $4 \mathrm{~g}$ of lyophilized $R$. marinus, with the above-mentioned purity.

The most widely used criterion to check biliprotein solution purity is the absorbance ratio $A_{\max }$ visible $/ A_{280}$; nevertheless, the purity must be confirmed using other experimental methods. Spectroscopic analyses of the fractions from size-exclusion chromatography were carried out. We also measured fluorescence emission spectra of purified B-PE that showed a maximum at $578 \mathrm{~nm}$ (fig. 4).

The B-PE subunits by SDS-PAGE showed two bands (fig. 5) containing closely similar amounts of protein. By comparison with standards, the molecular masses were 27000 and 26800 , corresponding to the $\alpha$ and $\beta$ subunits of B-PE.

Circular dichroism analysis was performed to fractionate with $\mathrm{B}-\mathrm{PE}$ to obtain structural information at $\mathrm{pH} 7$ and $\mathrm{pH} 2$. It is well known (Berova et al.1981) that $\alpha$ helix structures show an intense positive band at about $192 \mathrm{~nm}$ and two negative

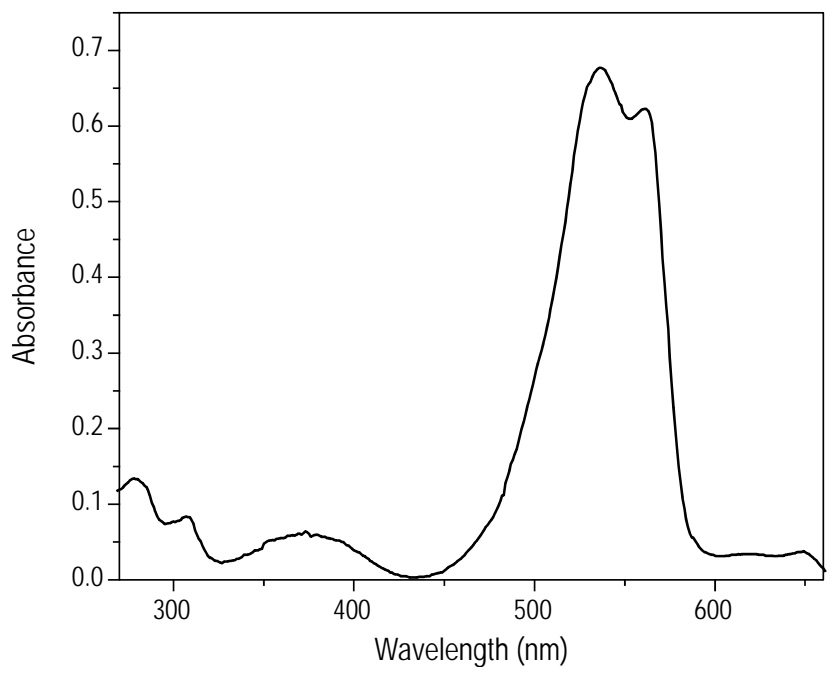

Figure 3. Absorption spectrum of B-phycoerythrin purified by sizeexclusion chromatography in HPLC.

Figura 3. Espectro de absorción de la B-ficoeritrina purificada mediante cromatografía de exclusión por tamaño (cromatografía líquida de alta resolución).

Las fracciones conjuntas obtenidas mediante elución con fosfato $0.01 \mathrm{M} / \mathrm{NaCl} 0.1 \mathrm{M}$ por cromatografía aniónica se introdujo en una columna para cromatografía de exclusión por tamaño. Los resultados obtenidos por HPLC mostraron una proteína con una pureza de $4.8\left(\mathrm{~A}_{545} / \mathrm{A}_{280}\right)$, adecuada para los pasos de purificación (fig. 3). La razón de la absorción a 545 $\mathrm{nm}$ entre la absorción a $498 \mathrm{~nm}\left(\mathrm{~A}_{545} / \mathrm{A}_{498}\right)$ fue 2.5; este valor es aceptable para la identificación del pigmento de ficoeritrina. Se obtuvieron alrededor de $1.292 \mathrm{mg}$ de B-PE de $4 \mathrm{~g}$ de $R$. marinus liofilizada, con la pureza ya mencionada.

El criterio utilizado con mayor frecuencia para determinar la pureza de la solución de biliproteínas es la razón de absorción $\mathrm{A}_{\text {max visible }} / \mathrm{A}_{280}$; no obstante, se debería de confirmar la pureza con otros métodos experimentales. Se realizaron análisis espectroscópicos de las fracciones de la cromatografía de exclusión por tamaño. También se midieron espectros de emisión de fluorescencia de la B-PE purificada que mostraron un máximo a $578 \mathrm{~nm}$ (fig. 4).

Mediante la SDS-PAGE las subunidades de B-PE mostraron dos bandas (fig. 5) que contenían cantidades similares de proteína. Al comparar con estándares, las masas moleculares fueron 27000 y 26800 , correspondiendo a las subunidades $\alpha$ y $\beta$ de B-PE.

Se realizó un análisis de dicroísmo circular para fraccionar con B-PE y obtener información estructural a pH 7 y pH 2. Es bien conocido (Berova et al. 1981) que las estructuras de hélice $\alpha$ muestran una banda positiva intensa alrededor de $192 \mathrm{~nm}$ y dos bandas negativas alrededor de 208 y $222 \mathrm{~nm}$. En la figura 6 se observa el comportamiento de la proteína a $\mathrm{pH} 7$ y pH 2 en el intervalo de 180 a $260 \mathrm{~nm}$. La curva muestra una banda positiva intensa alrededor de $195 \mathrm{~nm}$, la cual disminuyó cuando la proteína se encontraba en condiciones ácidas. Las bandas 


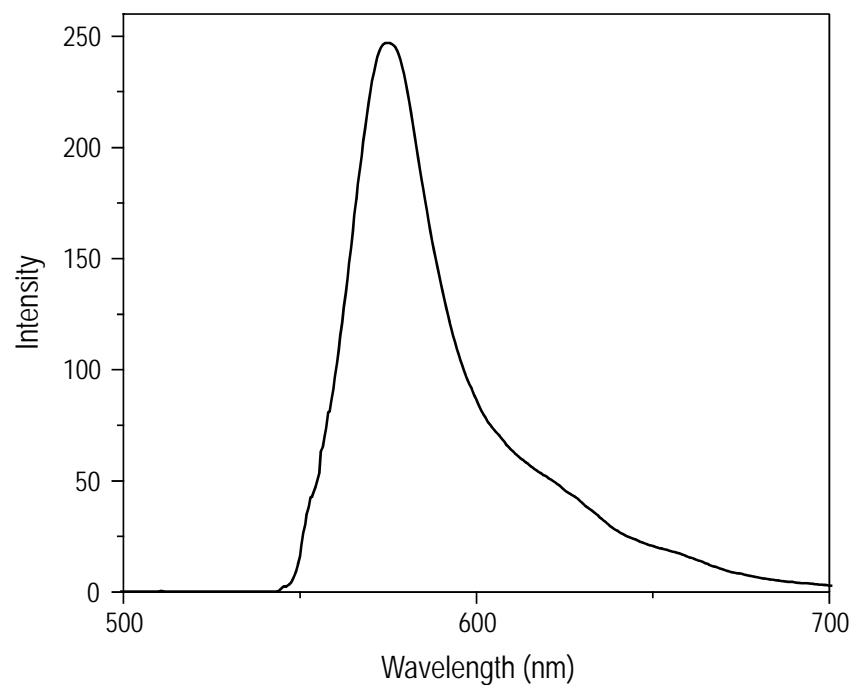

Figure 4. Visible fluorescence emission spectra of purified B-phycoerythrin. Figura 4. Espectros visibles de emisión de fluorescencia de la B-ficoeritrina purificada.

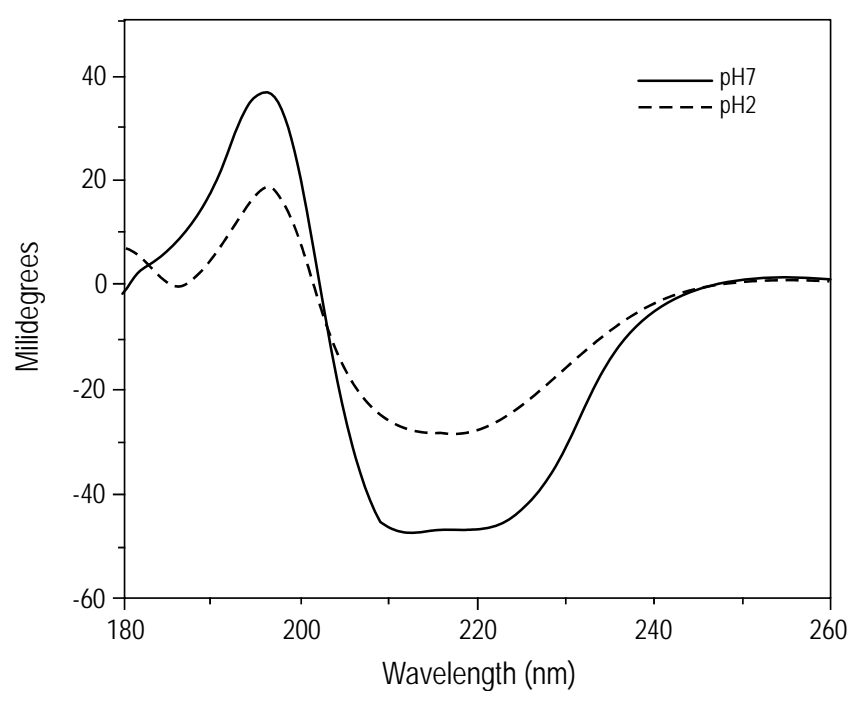

Figure 6. Far UV circular dichroism spectra of B-phycoerythrin in $0.01 \mathrm{M}$ Na-phosphate buffer at $\mathrm{pH} 7$ and $\mathrm{pH}$ 2. Protein concentration was $0.2 \mathrm{mg}$ $\mathrm{mL}^{-1}$.

Figura 6. Espectros de dicroísmo circular en el lejano UV de B-ficoeritrina en amortiguador de fosfato de sodio $0.01 \mathrm{M} \mathrm{a} \mathrm{pH} 7$ y pH 2. La concentración de proteína fue $0.2 \mathrm{mg} \mathrm{mL}^{-1}$.

bands at about 208 and $222 \mathrm{~nm}$. Figure 6 shows the behavior of the protein at $\mathrm{pH} 7$ and $\mathrm{pH} 2$ in the range of $180-260 \mathrm{~nm}$. As can be observed the curve shows an intense positive band at about $195 \mathrm{~nm}$, which decreased when the protein was under acidic conditions. The negative bands at 210 and $222 \mathrm{~nm}$ are present in the fraction at $\mathrm{pH} 7$, but the intensity of the bands decreased at $\mathrm{pH}$ 2. The results of the DICHROWEB analysis are shown in table 1, where the CDSSTR program was used to obtain the curve that best fits to the data.

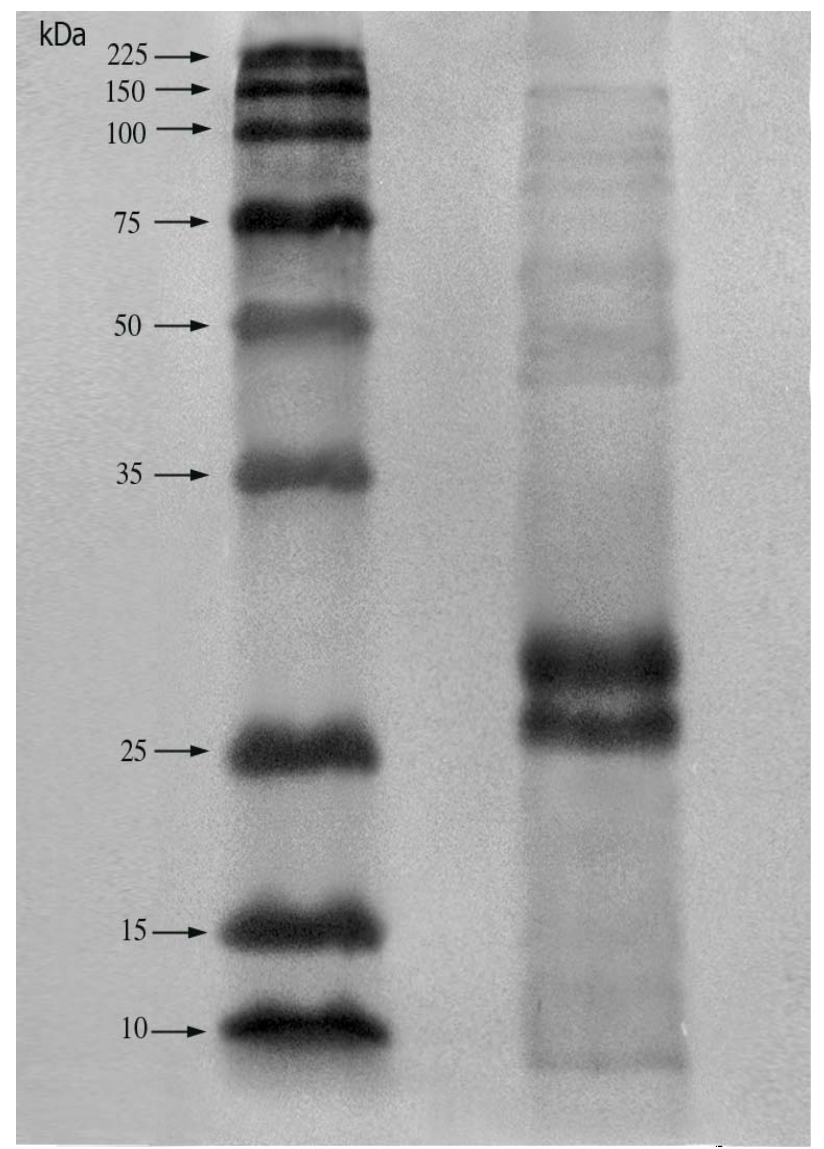

Figure 5. SDS-PAGE of purified B-phycoerythrin. From left to right the bands correspond to marker proteins and purified B-phycoerythrin after HPLC.

Figura 5. SDS-PAGE de la B-ficoeritrina purificada. De izquierda a derecha las bandas corresponden a proteínas marcadoras y B-ficoeritrina purificada después de la cromatografía líquida de alta resolución.

negativas a 210 y $222 \mathrm{~nm}$ se observan en la fracción a $\mathrm{pH} 7$, pero la intensidad disminuyó a pH 2. En la tabla 1 se muestran los resultados del análisis con DICHROWEB, en donde se usó el programa CDSSTR para determinar la curva que mejor se ajusta a los datos.

\section{Discusión}

Rhodosorus marinus presentó tres tipos de ficobiliproteínas: ficoeritrina, ficocianina y aloficocianina. Para la microalga roja Porphyridium cruentum, Bermejo et al. (2002) encontraron que el contenido de aloficocianina era insignificante o inexistente en comparación con la R-ficocianina y la B-PE.

En el presente trabajo se obtuvo la elución de la mayor parte de B-PE con fosfato de sodio $0.01 \mathrm{M} / \mathrm{NaCl} 0.1 \mathrm{M}$. Rossano et al. (2003) utilizaron la misma fuerza iónica para la elución de R-ficoeritrina con un amortiguador que contenía fosfato de sodio $0.05 \mathrm{M} / \mathrm{NaCl} 0.1 \mathrm{M}(\mathrm{pH} 7.0)$ en una columna de hidroxiapatita y fosfato de sodio $0.01 \mathrm{M} / \mathrm{NaCl} 0.1 \mathrm{M}$ 
Table 1. Secondary structure of B-phycoerythrin (B-PE). The software used was DICHROWEB.

Tabla 1. Estructura secundaria de la proteína B-ficoeritrina (B-PE). Se utilizó la paquetería DICHROWEB.

\begin{tabular}{cccccccc}
\hline B-PE & Helix 1 & Helix 2 & Strand 1 & Strand 2 & Turns & Unordered & Total \\
\hline pH 7 & 0.42 & 0.08 & 0.15 & 0.11 & 0.06 & 0.19 & 1.01 \\
pH 2 & 0.045 & 0.10 & 0.11 & 0.12 & 0.06 & 0.17 & 1.01 \\
\hline
\end{tabular}

\section{Discussion}

Rhodosorus marinus showed three types of phycobiliproteins: phycoerythrin, phycocianin, and allophycocyanin. Bermejo et al. (2002) showed that allophycocyanin content is negligible or absent relative to R-phycocyanin and B-PE in the red microalga Porphyridium cruentum.

In our purification, the majority of B-PE was eluted with $0.01 \mathrm{M}$ Na-phosphate/0.1 M NaCl buffer. The same ionic strength was used by Rossano et al. (2003), who eluted Rphyco-erythrin with a buffer containing $0.05 \mathrm{M} \mathrm{Na-phosphate/}$ $0.1 \mathrm{M} \mathrm{NaCl}(\mathrm{pH} 7.0)$ on a hydroxyapatite column and $0.01 \mathrm{M}$ Na-phosphate/0.1 M NaCl (pH 7.0) on a Superdex 75 gel filtration column. In our experiments we found that it was necessary to use size-exclusion chromatography following anionic chromatography to obtain highly purified B-PE.

The purified B-PE of $R$. marinus showed two peaks at 540 and $562 \mathrm{~nm}$, and a small shoulder at $498 \mathrm{~nm}$. It is known that there are two types of phycoerythrin in P. crenum: one is B-PE, which has an absorption shoulder at $498 \mathrm{~nm}$, and the other is b-phycoerythrin, which does not. We believe our preparations could have a mixture of both pigments because the shoulder is slight. In addition, there is only one emission peak at $578 \mathrm{~nm}$ but this is the same in both pigments. The fluorescence emission spectrum of the purified B-PE maximum is $578 \mathrm{~nm}$. This result is consistent with that published for the fluorescence spectrum of phycoerythrins (Bermejo et al. 2002).

We produced a B-PE with a purity index of $4.8\left(\mathrm{~A}_{545} / \mathrm{A}_{280}\right)$, which exceeds the standard purification of phycobiliproteins from other sources (Siegelman and Kycia 1978). The $\mathrm{A}_{545} / \mathrm{A}_{498}$ ratio was 2.5 , an acceptable value for identifying the phycoerythrin pigment as from typical B-PE (Gantt and Lipschultz 1974).

About $1.292 \mathrm{mg}$ of B-PE were obtained from $4 \mathrm{~g}$ of lyophilized $R$. marinus. Rossano et al (2003) obtained $15 \mathrm{mg}$ of pure phycoerythrin from $25 \mathrm{~g}$ of lyophilized Corallina elongata, with a similar $\mathrm{A}_{545} / \mathrm{A}_{280}$ ratio of 5 .

The SDS-PAGE results revealed two close intense bands at 26.8 and $27.7 \mathrm{kDa}$, whereas we expected 17 and $18 \mathrm{kDa}$, respectively, for the $\alpha$ and $\beta$ subunits found in the major cyanobacterial and red algal phycobiliproteins (phycoerythrin, phycocyanin, and allophycocyanin; Swanson and Glazer 1990). Both R- and B-PE have a $(\alpha \beta) 6 \gamma$ composition (Redlinger and Gantt 1981). Moreover, in the SDS-PAGE of $P$. cruentum, the B-PE shows the presence of at least three $\gamma$ subunits, differing slightly in molecular weight. We think what this
(pH 7.0) en una columna de filtración sobre gel Superdex 75. En nuestros ensayos fue necesario usar comatografía de exclusión por tamaño después de la cromatografía de intercambio aniónico para obtener B-PE altamente purificada.

La B-PE purificada de $R$. marinus mostró dos máximos, a 540 y $562 \mathrm{~nm}$, con un hombro pequeño a $498 \mathrm{~nm}$. Se sabe que existen dos tipos de ficoeritrina en $P$. crenum: una es B-PE, que tiene un hombro de absorción a $498 \mathrm{~nm}$, y la otra es b-ficoeritina, que no lo tiene. Creemos que nuestras preparaciones podrían tener una mezcla de ambos pigmentos ya que el hombro es pequeño. Además, sólo hay un pico de emisión a $578 \mathrm{~nm}$ pero es igual en ambos pigmentos. El espectro de emisión de fluorescencia del máximo de la B-PE purificada es $578 \mathrm{~nm}$. Este resultado concuerda con lo publicado para el espectro de fluorescencia de las ficoeritrinas (Bermejo et al. 2002).

En este estudio se obtuvo B-PE con un índice de pureza de $4.8\left(\mathrm{~A}_{545} / \mathrm{A}_{280}\right)$, que excede la purificación estándar de ficobiliproteínes de otras fuentes (Siegelman y Kycia 1978). La razón $\mathrm{A}_{545} / \mathrm{A}_{498}$ fue 2.5, un valor aceptable para identificar el pigmento de ficoeritrina como proveniente de B-PE típica (Gantt y Lipschultz 1974).

Se obtuvieron alrededor de $1.292 \mathrm{mg}$ de B-PE a partir de $4 \mathrm{~g}$ de $R$. marinus liofilizada. Rossano et al (2003) obtuvieron $15 \mathrm{mg}$ de ficoeritrina pura de $25 \mathrm{~g}$ de Corallina elongata liofilizada, con una razón $\mathrm{A}_{545} / \mathrm{A}_{280}$ similar, de 5 .

Los resultados de SDS-PAGE mostraron dos bandas cercanas intensas a 26.8 y $27.7 \mathrm{kDa}$, cuando se esperaban $17 \mathrm{y}$ $18 \mathrm{kDa}$, respectivamente, para las subunidades $\alpha$ y $\beta$ encontradas en las principales ficobiliproteínas de cianobacterias y algas rojas (ficoeritrina, ficocianina y aloficocianina; Swanson y Glazer 1990). Tanto la R-ficoeritrina como la B-PE tienen una composición $(\alpha \beta) 6 \gamma$ (Redlinger y Gantt 1981). Además, en la SDS-PAGE de P. cruentum, la B-PE muestra la presencia de al menos tres subunidades $\gamma$ que difieren ligeramente en peso molecular. Pensamos que esto le pudiese estar pasando a $R$. marinus ya que los resultados de SDS-PAGE muestran bandas difusas en la región de $\gamma$.

El análisis de dicroísmo circular mostró una intensa banda positiva alrededor de los $195 \mathrm{~nm}$ que decreció cuando la proteína se encontraba en condiciones ácidas. Esto podría indicar que también hay sábanas $\beta$ presentes en la estructura. Las bandas negativas a 210 y $222 \mathrm{~nm}$ se encuentran en la fracción a $\mathrm{pH} 7$, pero la intensidad de las bandas decreció a pH 2 debido a la pérdida de la estructura secundaria. Bermejo et al. (2001) observaron este fenómeno en la B-PE de P. cruentum, pero no estimaron su contenido de sábanas $\alpha$ y $\beta$. 
could be happening to $R$. marinus because the SDS-PAGE results show diffuse bands in the region of $\gamma$.

Circular dichroism analysis showed an intense positive band at about $195 \mathrm{~nm}$ that decreased when the protein was under acidic conditions. This could mean that $\beta$ sheets are also present in the structure. The negative bands at 210 and $222 \mathrm{~nm}$ are present in the fraction at $\mathrm{pH} 7$ but the intensity of the bands decreased at $\mathrm{pH} 2$ due to a loss of secondary structure. This phenomenon was also observed by Bermejo et al. (2001) with B-PE obtained from P. cruentum; however, they did not estimate the $\alpha$ and $\beta$ sheet content.

Secondary structure in B-PE from $R$. marinus does not appear to be greatly different at $\mathrm{pH} 2$ compared with $\mathrm{pH} 7$ (see table 1).

\section{Acknowledgements}

This research was supported by CONACYT (Mexico) (grant SEP-2004-C01-46749 and grant 83191). The first author acknowledges receipt of a scholarship from CONACYT.

\section{References}

Ayyagari M, Pande R, Kamtekar S, Gao H. 1995. Molecular assembly of proteins and conjugated polymers: Toward development of biosensors. Biotechnol. Bioeng. 45: 116-121.

Básaca-Loya A, Burboa MG, Valdez MA, Gámez R, Goycoolea FM, Gutiérrez-Millán LE. 2008. Aggregation behavior and rheology of culture broths of Rhodosorus marinus. Rev. Mex. Fís. S 54: $119-126$

Benavides J, Rito-Palomares M. 2004. Bioprocess intensification: A potential aqueous two-phase process for the primary recovery of B-phycoerythrin from Porphyridium cruentum. J. Chromatogr. B 807: 33-38.

Bermejo R, Talavera EM, Alvarez-Pez JM, Orte JC. 1997. Chromatographic purification of phycobiliproteins from Spirulina platensis. High-performance liquid chromatographic separation of their alfa and beta subunits. J. Chromatogr. A 778: 441-450.

Bermejo R, Talavera EM, Alvarez-Pez JM. 2001. Chromatographic purification and characterization of B-phycoerythrin from Porphyridium cruentum. Semipreparative high-performance liquid chromatographic separation and characterization of its subunits. J. Chromatogr. A 917: 135-145.

Bermejo R, Alvarez-Pez JM, Acién-Fernández FG, Molina E. 2002. Recovery of pure B-phycoerythrin from the microalga Porphyridium cruentum. J. Biotechnol. 93: 73-85.

Bermejo R, Acién FG, Ibáñez MJ, Fernández JM. 2003. Preparative purification of B-phycoerythrin from the microalga Porphyridium cruentum by expanded-bed adsorption chromatography. J. Chromatogr. B 790: 317-325.

Berova N, Nakanishi K, Woody RW (eds.). 1981. Circular Dichroism, Principles and Applications. 2nd ed. Wiley-VCH, New York.

Bradford MM. 1976. A rapid and sensitive method for the quantitation of microgram quantities of protein utilizing the principle of protein-dye binding. Anal. Biochem. 72: 248-254.

D’Agnolo E, Rizzo R, Paoleti S, Murano E. 1994. R-phycoerythrin from the red alga Gracilaria longa. Phytochemistry 35: 693-696.

Duerring M, Schmidt GB, Huber R. 1991. Isolation, crystallization, crystal structure analysis and refinement of constitutive Cphycocyanin from the chromatically adapting cyanobacterium
La estructura secundaria de la B-PE de $R$. marinus no parece ser muy diferente a $\mathrm{pH} 2$ al compararla con la estructura a pH 7 (ver tabla 1).

\section{Agradecimientos}

Este trabajo fue apoyado por el CONACYT (México) (convenio SEP-2004-C01-46749 y convenio 83191). La primera autora recibió una beca del CONACYT.

Traducido al español por Christine Harris.

Fremyella diplosiphon at $1.66 \AA$ resolution. J. Mol. Biol. 217: 577-592.

Ficner R, Lobeck K, Schmidt G, Huber R. 1992. Isolation, crystallization, crystal structure analysis and refinement of Bphycoerythrin from the red alga Porphyridium sordidum at $2.2 \AA$ resolution. J. Mol. Biol. 228: 935-950.

Gantt E. 1975. Phycobilisomes: Light harvesting pigment complex. BioScience 25: 781-787.

Gantt E, Lipschultz CA. 1974. Phycobilisome of Porphyridium cruentum: Pigment analysis. Biochemistry 13: 2960-2966.

Glazer AN. 1981. Photosynthetic accessory proteins with bilin prosthetic groups. In: Conn EE, Stumpf PK (eds.), The Biochemistry of Plants. Vol. 8. Academic Press, New York, pp. 51-96.

Glazer AN. 1984. Phycobilisome: A macromolecular complex optimized for light energy transfer. Biochem. Biophys. Acta 768: $29-51$.

Glazer AN. 1989. Light guides. Directional energy transfer in a photosynthetic antenna. J. Biol. Chem. 264: 1-4.

Glazer AN, Hixson CS. 1975. Characterization of R-phycocyanin. J. Biol. Chem. 250: 5487-5495.

Grabowski J, Gantt E. 1978. Photophysical properties of phycobiliproteins from phycobilisomes: Fluorescence life-times, quantum yields and polarization spectra. Photochem. Photobiol. 28: 39-45.

Gray BH, Gantt E. 1975. Spectral properties of phycobilisomes and phycobiliproteins from the blue-green alga Nostoc spp. Photochem. Photobiol. 21: 121-128.

Grossman AR. 1990. Chromatic adaptation and the events involved in phycobilisome biosynthesis. Plant Cell Environ. 13: 651-666.

Haugland RP. 1996. Handbook of Fluorescent and Research Chemicals. 6th ed. Molecular Probes, Eugene, Oregon.

Hilditch CM, Balding P, Jenkins R, Smith AJ. 1991. R-phycoerythrin from the macroalga Corallina officinalis (Rhodophyceae) and application of a derived phycofluor probe for detecting sugarbinding sites on cell membranes. J. Appl. Phycol. 3: 345-354.

Holzwarth AR. 1991. Structure function relationship and energy transfer in phycobiliprotein antennae. Plant Physiol. 83: 518-528.

Iijima N, Shimamatsu H. 1982. Antitumor agent and method of treatment therewith. US Pat. Pending Ref. p1150-726-A82679.

Laemmli UK. 1970. Cleavage of structural proteins during the assembly of the head of bacteriofage T4. Nature 277: 680-685.

Ma SY, Wang GC, Sun HB, Zeng CK. 2003. Characterization of the artificially covalent conjugate of B-phycoerythrin and Rphycocyanin and the phycobilisome from Porphyridium cruentum. Plant Sci. 164: 253-257.

MacColl R, Guard-Friar D. 1983. Phycocianin 645: The chromophore assay of phycocyanin 645 from the cryptomonad protozoa Chroomonas species. J. Biol. Chem. 258: 14324-14329.

Moerschel E. 1991. The light-harvesting antennae of cyanobacteria and red algae. Photosynthesis 25: 137-144. 
Redlinger T, Gantt E. 1981. Polarized photo acustic spectra of phycobilisomes in ployvinyl alcohol films. Plant Physiol. 68: 1375-1379.

Rosowski JR, Parker BC (eds). 1971. Selected Papers in Phycology. Dept. of Botany, Univ. of Nebraska, Lincoln, 876 pp.

Rossano R, Ungaro N, D’Ambrosio A, Liuzzi GM. 2003. Extracting and purifying R-phycoerythrin from Mediterranean red algae Corallina elongate Ellis \& Solander. J. Biotechnol. 101: 289-293.

Schoelember RW, Leung S, Lundell D, Glazer AN. 1983. Chromopeptides from phycoerythrins. Structure and linkage of a phycoerythrobilin tryptic tripeptide derived from a Bphycoerythrin. J. Am. Chem. Soc. 105: 4072-4076.

Siegelman HW, Kycia JH. 1978. Algal biliproteins. In: Hellebust JA, Cragie JS (eds.), Handbook of Phycological Methods. Physiological and Biochemical Methods. Cambridge Univ. Press, pp. $71-79$.
Swanson RV, Glazer AN. 1990. Separation of phycobiliprotein subunits by reverse-phase high-pressure liquid chromatography. Anal. Biochem. 188: 295-299.

Tchernov AA, Minkova KM, Houbavenska NB, Kovacheva NG. 1999. Purification of phycobiliproteins from Nostoc sp. by aminohexyl-sepharose chromatography. J. Biotechnol. 69: 69-73.

Wang GC, Zhou BC, Tseng CK. 1998. Isolation, properties and spatial site analysis of the $\gamma$ subunits of B-phycoerythrin and Rphycoerythrin. Sci. China (Ser. C) 41(1): 9-17.

Yu MH, Glazer AN, Spencer KG, West JA. 1981. Phycoerythrins of the red alga Callithamnion. Plant Physiol. 68: 482-488.

Recibido en mayo de 2009;

aceptado en octubre de 2009. 